\title{
Single Particle Analysis: Methods for Detection of Platelet Extracellular Vesicles in Suspension (Excluding Flow Cytometry)
}

\author{
Edit I. Buzás ${ }^{1,{ }^{*}}$, Chris Gardiner ${ }^{2,}$, , Changwon Lee ${ }^{3,},{ }^{*}$ Zachary J. Smith ${ }^{4,}{ }^{*}$ \\ ${ }^{1}$ Department of Genetics, Cell- and Immunobiology, Semmelweis University, \\ Budapest, Hungary, edit.buzas@gmail.com \\ ${ }^{2}$ Department of Hematology, University College London, London, United Kingdom, \\ c.gardiner@ucl.ac.uk \\ ${ }^{3}$ Center for Biophotonics, University of California Davis Medical Center, Sacramento, \\ CA, USA, 95817, chwlee@ucdavis.edu \\ ${ }^{4}$ Department of Precision Machinery and Precision Instrumentation, University of \\ Science and Technology of China, Hefei, Anhui, China, 230027, zsmith@ustc.edu.cn \\ ${ }^{*}$ All authors contributed equally to this manuscript
}

\begin{abstract}
Extracellular vesicles (EVs) are small membrane-bound particles released by all cell types, including abundant release by platelets. EVs are a topic of increasing interest in the academic and clinical community due to their increasingly recognized and diverse role in normal biology as well as in disease. However, typical analysis methods to characterize EVs released by cell culture or isolated from whole blood or other body fluids are restricted to bulk analysis of all EVs in a sample. In this review we discuss the motivation for analysis of individual EVs, as well as discuss three emerging methods for physical and chemical characterization of individual EVs: Nanoparticle Tracking Analysis, Tunable Resistive Pulse Sensing, and Raman Spectroscopy. We give brief descriptions of the working principles of each technique, along with a review noting the benefits and limitations of each method as applied to detection of single EVs.
\end{abstract}

\section{Introduction \& Motivation}

Extracellular vesicles (EVs) are sub-micron membrane-enclosed vesicles that are released from all cell types. EVs play key roles in many physiological and pathological processes and are an important mode of intercellular communication through the transfer of nucleic acids (principally RNA species) and proteins. The protein and RNA content of EVs is dependent on the cellular origin and the physiological and pathological processes of the parent cell. Consequently, EVs have great potential as biomarkers of disease and as novel targets for therapeutic intervention ${ }^{1}$.

The measurement and characterisation of EVs presents unique problems. Most EV are 50 to $200 \mathrm{~nm}$ in diameter and have a low refractive index, i.e., they scatter very little light ${ }^{2,3}$. This presents problems for detection by light scattering methods and the majority of EVs are undetectable by conventional flow cytometry. EVs are often polydisperse in size, ranging from $50 \mathrm{~nm}$ to over $1000 \mathrm{~nm}$ in diameter. Interference from lipoproteins ${ }^{4}$ and protein aggregates ${ }^{5}$ of similar size adds to the complexity. The 
scarcity and unpredictable expression of antigens makes labelling difficult. Antigen density on EVs is frequently low and expression of an antigen on the parent cell is no guarantee that the same antigen will be present on EVs released from that cell ${ }^{6}$. Furthermore, "typical" EV antigens (e.g., CD63) are not present on all EVs.

Platelet-derived EVs (PEVs) were first described by Wolf ${ }^{7}$ as "platelet dust" with procoagulant activity in platelet poor plasma. Platelets release two types of vesicles: microvesicles (MV) (also referred to as microparticles or ectosomes) which are 100 $\mathrm{nm}$ to $1000 \mathrm{~nm}$ in diameter and bud directly from the plasma membrane; and exosomes which are typically smaller (generally considered to be $50 \mathrm{~nm}-150 \mathrm{~nm}$, but may be larger) and are released via multivesicular bodies ${ }^{8}$. It has been widely reported, and has been generally accepted, that PEVs are the most abundant type of circulating EV in the bloodstream, with estimates of 70-90\% widely reported $6,9,10$. It was also thought that PEVs predominantly exposed phosphatidylserine (PS), and annexin V labelling was frequently used to identify EVs by flow cytometry ${ }^{11}$. This is because the origin of PEV was assumed to be activated platelets, as isolated platelets released a large number of PEVs when activated.

However, it has been demonstrated that substantial amounts of PEVs (and non-PEVs as well) are generated in vitro artifactually in the blood collection tube, and acidcitrate dextrose (ACD-A) tubes proved to be the most efficient in preventing this in vitro vesiculation ${ }^{12}$. Furthermore, recent studies using cryo-electron microscopy ${ }^{13}$ and high sensitivity flow cytometry ${ }^{14}$ have shown that PEV may account for only 20 to $30 \%$ of total EVs in normal plasma. It is notable that in these studies, many EVs isolated from plasma have no detectable cell-specific antigen at all. These 'orphan' EVs are predominantly $<200 \mathrm{~nm}$ in diameter ${ }^{15}$ and it is highly probable that cellspecific antigens are present but at a level below the limit of detection of flow cytometry. However, it is wrong to assume that, because the majority of large EVs are platelet derived, these orphan EVs have the same origin. Conversely, our inability to detect platelet markers on the smallest plasma EVs should not be taken as proof of absence. It is clear that improved labelling techniques are required to identify the cellular origin of the small unlabelled EVs. Lipoproteins (such as low density lipoprotein (LDL) particles) have been shown recently to mimic circulating EVs, and also to attach to the surface of EVs (possibly hindering EV markers) ${ }^{4}$.

It is now known that the majority of PEVs isolated from normal plasma do not bind annexin $V^{13,14,16}$. This is in contrast to PEVs shed by ionophore treated platelets, the majority of which expose sufficient PS to bind annexin $V^{14}$. Furthermore, in healthy individuals, most circulating CD41 positive do not express platelet activation markers, e.g., CD63 and CD62P. These CD41+/CD62P- EVs lack LAMP-1 (lysosomeassociated membrane glycoprotein-1) but express full length filamin $A$, and are derived directly from megakaryocytes. In contrast, CD41+/CD62P+EVs are LAMP-1 positive and do not express full length filamin $\mathrm{A}^{17}$. This may explain the difference between the proteome of plasma -derived EVs and EVs shed by activated platelets ${ }^{18}$. 
It has long been known that PEVs have an important role in both normal haemostasis and thrombus formation. PEVs also play a key role in inflammation, atherosclerosis, neurodegenerative disorders, cancer progression and metastasis, angiogenesis and wound healing ${ }^{19}$. PEVs are a rich source of circulating miRNA ${ }^{20}$ and can deliver miRNA to endothelial cells ${ }^{21}$, macrophages ${ }^{22}$ and tumour cells ${ }^{23}$ resulting in altered gene expression. Consequently the study of PEVs and their effect on target cells will provide information on their important role as biomarkers or potential therapeutic agents.

However, given the wide apparent variability in the physical properties (size, surface charge, refractive index) and chemical properties (surface marker expression, intravesicle cargo, etc.), bulk analyses of "PEVs" are not sufficient to characterize vesicle populations. For that reason, increasing emphasis has been placed on developing instrumentation and measurement methodologies allowing researchers to characterize these chemical and physical properties on vesicle by vesicle basis. In this review we describe the physical mechanisms underlying these emerging methods along with representative results.

\section{Nanoparticle Tracking Analysis}

Nanoparticle tracking analysis (NTA) is a method for visualising and characterising particles suspended in a liquid. During analysis, particles in suspension are illuminated by a focused laser beam and light is scattered in all directions by the particles. Although particles smaller than $200 \mathrm{~nm}$ in diameter are below the limit of resolution of light microscopy, scattered light collected at 90 degrees relative to the laser is focussed onto an image sensor of a camera, allowing the visualisation of the position of the particles in suspension. A useful analogy is that dust particles, too small to be seen with the naked eye, can be "seen" when they scatter sunlight.

Nanoparticles suspended in a liquid move in Brownian motion at a rate determined by the viscosity of the liquid, the temperature of the liquid and the size of the particle, as shown schematically in Figure 1. Thus, if the "speed" (or mean square displacement) of a particle's Brownian motion (the diffusion coefficient) and the viscosity and temperature of the liquid are known, the hydrodynamic radius of the particle may be calculated using the Stokes-Einstein equation.

$D_{t}=\frac{K_{B} T}{3 \pi \eta d_{h}}$

$$
\begin{aligned}
& \mathrm{D}_{\mathrm{t}}=\text { diffusion coefficient }, \mathrm{K}_{\mathrm{B}}=\text { Boltzman constant }, \eta=\text { viscosity, } \\
& \mathrm{T}=\text { absolute temperature }, \mathrm{d}_{\mathrm{h}}=\text { hydrodynamic diameter }
\end{aligned}
$$

A video recording of particles is made (acquisition) and the video is then analysed by the NTA software. NTA identifies and tracks each individual nanoparticle from frame to frame, hence the mean square displacement can be measured, and from it the 
diffusion coefficient is calculated ${ }^{24}$. Furthermore, the intensity of light scattered by the particles may be calculated, and from this a refractive index of each vesicle may be determined ${ }^{3,25}$.

NTA is a useful method for the rapid (hundreds of EVs per minute) assessment of EV size, refractive index, and approximate concentration, as shown in Figure 2. It is most applicable to small EVs in the $50 \mathrm{~nm}-250 \mathrm{~nm}$ range which are below the detection limit of conventional flow cytometry ${ }^{26}$. Measurements are typically made in isotonic phosphate buffered saline (PBS) which preserves the size of EVs and, as PBS has the same viscosity as distilled water (the default diluent for NTA), eliminates the necessity for measurement of viscosity.

In order to measure EVs by NTA, the sample is diluted to approximately $1-10 \mathrm{x}$ $10^{8} / \mathrm{mL}$. It is preferable to make several short recordings (e.g. 10-30 recordings of 1030 seconds each), refreshing the sample between measurements to reduce sampling error and avoiding loss of EVs due to settling or adhesion ${ }^{27}$. The best camera level depends upon the size of the EVs. Ideally, each EV should be appear as a single point of light but this may be difficult to achieve if the EVs are highly polydisperse. For standard NanoSight systems (Malvern Instruments, UK) in earlier versions of NTA software, camera gain and shutter speed could be set using the advanced mode. In later versions, sixteen optimised camera levels are used ranging from level 1 (the least sensitive; gain 0 , shutter speed 1) to level 16 (the most sensitive; gain 512, shutter speed 1,300). The NTA software settings are: detection threshold, minimum expected particle size, blur, minimum track length, and background subtraction. In recent versions of NTA software, these settings are automated with the exception of detection threshold.

It is advisable to regularly check the performance of the instrument using microspheres of known size and concentration. In the past, most standardisation was performed using NIST-traceable polystyrene microspheres. However, polystyrene has a much higher refractive index (1.59) than EVs (Approximately 1.37 -1.42), so silica microspheres (refractive index 1.47) are a more appropriate material for standardising EV measurements ${ }^{3,25}$.

While the measurement of EV size and concentration can provide useful information, the ability to phenotype EVs using fluorescent labelling techniques is far more informative. Until now, single EV analysis using conventional antibody labelling has been largely restricted to the largest EVs of $>300 \mathrm{~nm}$ in diameter (approximately $1 \%$ ). Most EV are $50-200 \mathrm{~nm}$ in diameter and antigen expression is typically low. While some next-generation flow cytometers can detect EVs as small as $120 \mathrm{~nm}$, detection of specific antigens on small EVs has only been possible for a few highly expressed antigens. There are several reasons for this: the large size of IgG-fluorophore conjugates $(20-60 \mathrm{~nm})^{28}$ relative to EVs limits the number of molecules that can bind to a given EV; steric hindrance of IgG-antigen binding and antigen clustering further limit antigen detection; lastly, fluorophore conjugation is frequently unpredictable as the positioning of large fluorophores and the conjugation process itself may interfere 
with the antigen binding site. However, EVs generated from cells stably transfected with enhanced green fluorescent protein (EGFP) constructs are easily detectable using fluorescence NTA with a $488 \mathrm{~nm}$ laser source and a $500 \mathrm{~nm}$ long pass filter. Photon correlation spectroscopy showed that EVs expressing as few as 10 GFP molecules could be reliably detected by NTA (unpublished data). EGFP expressing EVs do not suffer from the background fluorescence associated with immunofluorescent or membrane labelling, so this level of sensitivity may not be achievable using these techniques. The problem lies not with the detection sensitivity of NTA but with the limitations of the labelling techniques. Direct ${ }^{26}$ and two stage labelling with quantum dots ${ }^{29}$ has been demonstrated but loss of antibody reactivity during conjugation, non-specific binding, quantum dot aggregation and background fluorescence have limited the successful application of this technique.

Monoclonal antibodies optimised for flow cytometry are a $20^{\text {th }}$ century solution to a $21^{\text {st }}$ century problem. There is a pressing need for extremely small, specific, high affinity fluorescently conjugated molecular probes for EV labelling. Fluorescently labelled aptamers, affibodies or nanobodies all offer potential solutions to specific EV labelling.

\section{Resistive Pulse Sensing}

Resistive pulse sensing, also known as the Coulter effect, describes the change in resistance between two electrodes as non-conducting particles pass through an aperture separating the electrodes, shown schematically in Figure 1. The electrodes are submerged in an electrolyte solution permitting a current to flow between them with a current drop across the electrodes due to the native resistance of the electrolyte solution. As particles pass through the aperture that separates the electrodes, they displace a certain volume of the electrolyte solution, changing the resistance of the circuit and causing an increasing drop in current, producing a "resistive pulse." The size of this pulse is linearly proportional to the volume of the particle passing through the aperture. Resistive pulse sensing is widely used in hematology for sizing blood cells and platelets in standard hematology analyzers. However, the smallest volume that can be accurately recorded with these devices depends on the aperture size and detection electronics. Standard hematology analyzers, for example, have only in recent years been able to reliably report platelet volumes.

A novel technology known as Tunable Resistive Pulse Sensing (TRPS) has recently been developed ${ }^{30}$ that permits accurate sizing and counting of very small particles with high accuracy by using a sub-micrometer pore poked in an elastic membrane. Due to the flexibility of the membrane, the pore itself can be dynamically adjusted, changing its size and conductive properties to best suit varying samples, or even to discriminate between different populations within a single experiment ${ }^{31}$. This technology provides a wealth of information about the particles passing through the

aperture. By precisely controlling the flow-rate of particles through the system, as well as by varying the flow and charge in the device and using calibration particles, 
one can determine particle size, particle count (or concentration), as well as charge characteristics (Zeta potential/electrophoretic mobility), as shown in Figure 2. Commercial TRPS systems generally have a size detection threshold of around $40 \mathrm{~nm}$, and an upper limit given by the pore size.

TRPS is a relatively new technology, and as such the majority of published studies are currently examining the proper techniques and protocols for quantifying EVs using TRPS. Several studies have been made comparing several methods to determine the applicability of TRPS to EVs, concluding that, broadly speaking, under proper conditions, TRPS provides similar quantification ability compared to NTA and flow cytometery 2,32-35. There is a single study examining the use of TRPS on quantifying platelet EVs, where their larger size compared with, for example, exosomes, makes them ideal targets for TRPS ${ }^{36}$. The relative simplicity and accuracy of TRPS measurements has helped it to rapidly transition to an accepted modality for characterization of EVs. For example, it was recently used as the standard method to evaluate different isolation procedures on exosome yield and size, ${ }^{37}$ and has been highlighted as an accepted characterization method in a ISEV position paper dating to $2012^{38}$. Nevertheless, as with all techniques, TRPS has its own limitations that have been called out by several studies, such as minimum detectable size ${ }^{32}$ and maximum throughput 35 that should be kept in mind. At higher throughputs (or with particles larger than the pore diameter), pore clogging can occur at unacceptable rates. Clogging requires the experiment to be paused to unclog the system, and the clog itself may alter the dimensions of the pore in unpredictable ways. ${ }^{34}$ Furthermore, while it characterizes physical parameters of EVs extremely well, it provides no information about chemical content, and there is no labelling technique to provide subpopulation specificity in TRPS measurements.

However, recently it has been shown that TRPS can be efficiently combined with differential detergent lysis ${ }^{39}$. The disappearance of a TRPS signal at a given concentration of a detergent not only proves the vesicular nature of a particle, but it is also characteristic for size-based EV subpopulations. EVs with small diameter $(\sim 100 \mathrm{~nm})$ are more resistant to detergent lysis than those with larger diameter (>150 nm) suggesting distinct membrane lipid composition (and possibly different biogenesis) of these EVs ${ }^{40}$.

\section{Raman Spectroscopy}

\section{4a. Spontaneous Raman Scattering}

Raman scattering is the inelastic scattering of light by individual molecules. When a light source is incident on a molecule, there is a (small) chance of inelastic scattering of the photon, where energy is transferred either to or from the molecule, giving rise to scattered photons with wavelengths shifted from the incident source. The wavelength shifts for a given molecule depend on the molecule's specific molecular 
arrangement, as shown in Figure 1. Thus, measuring the spectrum of these inelasticscattered photons, we obtain a "spectral fingerprint" for that molecule. Raman scattering can independently report on the chemical content of a wide variety of biomolecules in a single measurement with no need for any sample preparation or additional contrast agents. Due to these advantages, Raman spectroscopy is increasingly being explored as a fundamental analytical tool in biology and medicine ${ }^{41}$.

In Raman spectroscopy, the sample volume probed by the system is determined by the area illuminated with the laser beam, which can be less than $1 \mathrm{fL}$ for tightly focused beams. This tightly focused laser can also trap and confine micro- and nanoparticles and keep them at the focus of the laser beam for prolonged measurements. This combination of optical trapping and Raman spectroscopy is termed LaserTweezers Raman Spectroscopy (LTRS), and, since its introduction in the early 2000s, has been used in a wide range of single-cell and single-particle studies ${ }^{42-45}$.

The ability to trap and observe chemical information from individual particles makes it particularly attractive for studies of chemical heterogeneity of EVs. Yet, possibly due to the difficulty in obtaining Raman spectra from EVs, relatively few such studies exist in the literature. Early results focus on measuring bulk preparations of EVs ${ }^{46}$. Following this, Tatischeff et al. used LTRS to trap and record Raman spectra of small numbers of EVs (approximately 2-10) released by Dictyostelium discoideum, as well from human urine samples ${ }^{47}$. The authors observed consistent changes in the composition of the EVs depending on the growth state of the Dictyostelium organism. Furthermore, they also observed that EVs from human urine appear to vary slightly based on donor, potentially indicating something about the patient health status.

In 2015, Smith et al., recorded Raman spectra hypothesized to be from individual EVs extracted from a wide range of mammalian cell lines ${ }^{48}$.EVs from multiple cell lines grouped into four clusters with distinct biochemical composition. By analyzing the spectral features corresponding to each group, chemical differences were found to originate from membrane composition (cholesterol, lipid:cholesterol ratio, and surface protein expression). These results not only demonstrate the level of chemical detail afforded by Raman spectroscopy, but also highlighting the heterogeneity of vesicle composition even from the highly idealized samples of immortalized cell lines. This further underscores the importance of single-vesicle methods described in this review. Representative results of Raman spectra of vesicle samples are shown in Figure 2.

Despite Raman spectroscopy's unique advantages, it suffers from extremely long integration times (ranging from 1 to 5 minutes per vesicle), that limits its ability to be used in a clinical setting (as contrasted with basic biological studies). This places the throughput of spontaneous-Raman-based systems 4-5 orders of magnitude lower than methods such as NTA or TRPS. To overcome this drawback, some researchers have been exploring methods to increase the signal strength and overall throughput of Raman measurements of EVs, as described below. 


\section{4b: Surface Enhanced Raman Scattering (SERS)}

After the discovery of great enhancement of Raman scattering of pyridine molecules adsorbed on the surface of roughened silver in $1973^{49}$, Surface Enhanced Raman Spectroscopy (SERS) has been widely applied for the detection and analysis of biological samples including molecules, proteins and cells ${ }^{50,51}$. When light of an appropriate frequency interacts with the metallic structure, a surface plasmon (a collective oscillation of the electrons) forms and propagates on the surface of the metal. This gives rise to an enhanced electromagnetic field near the nanostructure depending on the structure of the nanomaterials. This enhanced electromagnetic field greatly intensifies the generated Raman scattering, with enhancement factors as ranging from $10^{6}$ to $10^{15}$ reported. Since it is a surface plasmon which excites the molecules for the SERS process, the sample of interest should be located within $5 \mathrm{~nm}$ from the surface of the metallic nanostructured substrate.

The SERS technique was applied for the rapid biochemical analysis of the vesicle surface contents including membranes and surface proteins. Kerr et al. reported SERS analysis on ovarian tumor derived EVs by mixing the EVs with gold nanoparticles ${ }^{52}$, while Tirinato et al. showed SERS spectra purportedly arising from EVs on super-hydrophobic nanopillars ${ }^{53}$. However, because the authors did not correct for the SERS signal of the isolation kit used in the study, a substantial portion of that signal is likely to be from that contaminant ${ }^{48,54}$, highlighting some of the difficulty in performing label-free measurements on chemically complex samples such as EVs. Lee et al., developed an efficient substrate system to capture and SERSanalyze EVs using nanobowl shaped planar, thin-metal coated substrates ${ }^{54}$. In this work, the authors have shown a simple, soft lithographic method for the substrate fabrication using flexible poly(dimethylsiloxane) (PDMS) templates on which a thin layer of silver is sputtered. The sputtered surface has a significantly lower background compared with SERS particles prepared via reduction methods. Results also suggest that the bowl-shaped substrate allows intact and ruptured EVs to be measured using time-dependent measurements, where spectra were measured at different time points as the sample solution dried on the substrate showed peak evolution over the extended time period.

Stremersch et al. describe coating individual exosome-like vesicles (ELVs) with $10 \mathrm{~nm}$ gold nanoparticles coated with 4-dimethylaminopyridine (DMAP) ${ }^{55}$. This coating gave the nanoparticles a strong surface charge that allowed electrostatic binding to the ELVs. After depositing a prepared sample on a quartz surface, the authors were able to record SERS signals from what are hypothesized to be individual ELVs, showing distinct spectral differences from ELVs obtained from two separate cell types. However, the strong background from the DMAP itself makes interpretation of the spectra difficult, highlighting an additional challenge of SERS measurements, where subtle changes in the nanostructure used to enhance the signal has a very strong impact on the recorded spectrum.

\section{Future Outlook \& Concluding Remarks}


Here we have presented several techniques capable of single-exosome analysis. Some, like Raman spectroscopy, remain largely unexplored, while others, such as NTA, have become standard methods in exosome analysis. However, even for standard techniques such as NTA, significant unanswered questions remain regarding preparation protocols and analysis methods. Furthermore, the increasing availability of optical labels such as fluorophores or highly Raman-active compounds that are conjugated to molecularly specific small molecules such as peptides, aptamers, etc., have the potential to continue to revolutionize the ease, specificity, and applicability of these methods to vesicle analysis.

The pressing need for single-vesicle analysis methods will also undoubtedly lead to the development of additional techniques not described here. For example, surface plasmon resonance (SPR) have recently been used to do detailed molecular profiling of extracellular vesicles 56,57 . While plasmon-based techniques currently are performed only on bulk samples, similar platforms have already been shown to have achieved sensitivity to single-molecule binding events ${ }^{58}$. Therefore, it is not difficult to envision single-exosome studies using these methods arriving in the near future.

Another promising method is a recently-developed microtoroid-based biosensor ${ }^{59}$. In this technology, nicknamed FLOWER (Frequency Locked Optical Whispering Evanescent Resonator) a tunable laser probes the resonant frequency of a single silica microtoroid coated with capture antibodies. As a sample is washed over the toroid, EVs with the appropriate surface markers bind to the toroid and with each binding event the resonant frequency shifts slightly. Thus FLOWER, similarly to NTA or TRPS is able to provide an accurate count of vesicle subpopulations, in addition to other physical parameters such as size and dry mass based on the magnitude of the change in resonant frequency upon binding.

The study of extracellular vesicles is still a nascent one, and thus researchers are forced to grapple simultaneously with critical biological questions and critical technological or practical questions arising due to the lack of standard techniques and protocols with long and established histories. Thus technologists and clinical or biological researchers alike must continue to work together to pave the way forward to precise, accurate, and repeatable characterization of these highly challenging and heterogeneous samples.

\section{Declaration of Interest Statement}

E. I. B., C. G. and C. L. report no conflicts of interest. Z.J.S. gratefully acknowledges funding support from the National Natural Science Foundation of China's 1000 Young Talents Plan. 


\section{Tables and Figures}

Table 1: A basic description of three methods for quantification of single vesicle characterization methods.

\begin{tabular}{|c|c|c|c|c|}
\hline Method & $\begin{array}{c}\text { Physical } \\
\text { Mechanism }\end{array}$ & $\begin{array}{c}\text { Supplied } \\
\text { Information }\end{array}$ & Throughput & $\begin{array}{c}\text { ISEV } \\
\text { Standard }\end{array}$ \\
\hline NTA & $\begin{array}{l}\text { Light scattering, } \\
\text { fluorescence }\end{array}$ & $\begin{array}{l}\text { Number, size, } \\
\text { refractive index, } \\
\text { surface marker }\end{array}$ & $6000 / \mathrm{min}$ & Yes \\
\hline TRPS & Coulter effect & Number, size, charge & $3000 / \mathrm{min}$ & Yes \\
\hline Raman & $\begin{array}{l}\text { Inelastic light } \\
\text { scattering }\end{array}$ & $\begin{array}{l}\text { Number, Chemical } \\
\text { makeup }\end{array}$ & $\begin{array}{c}\sim 0.2 / \mathrm{min} \\
\text { (spontaneous) }\end{array}$ & No \\
\hline
\end{tabular}

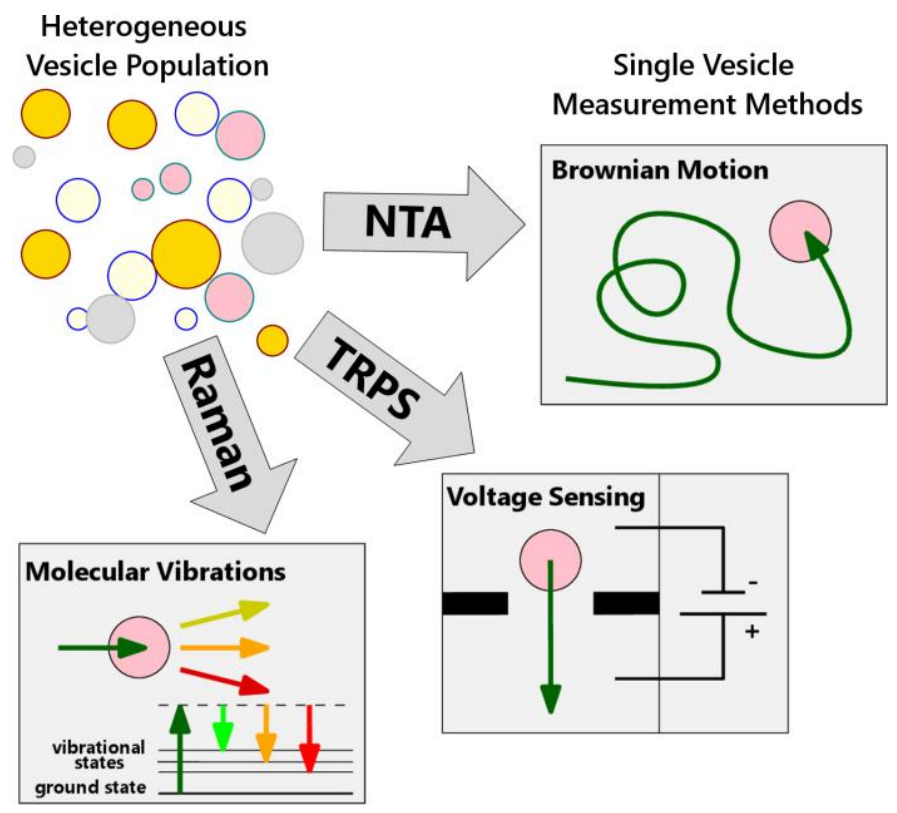

Figure 1: Schematic diagram of single-vesicle measurement methods. As described in detail in the text, vesicles of assorted size, internal contents, and membrane composition, are measured by imaging, voltage pulses, and optical spectroscopy. 

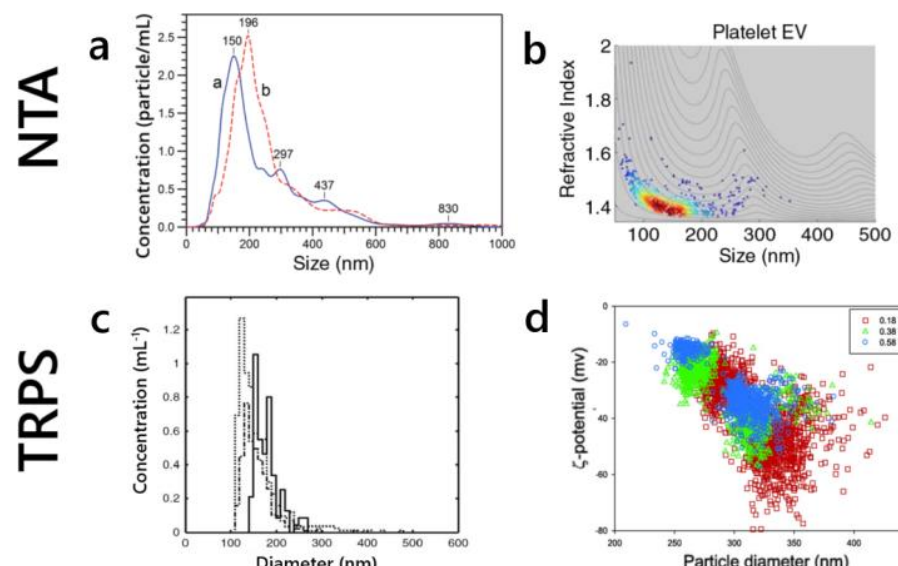

d
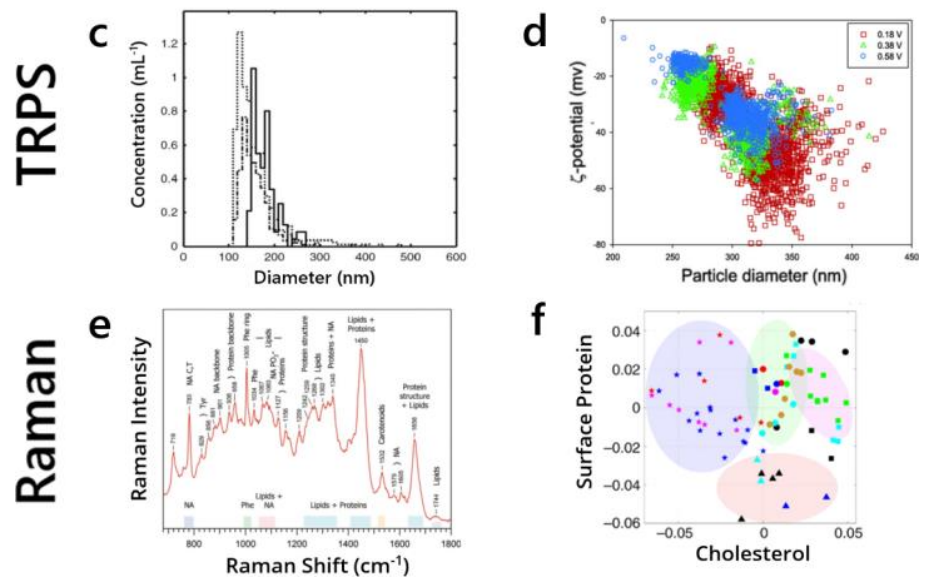

f

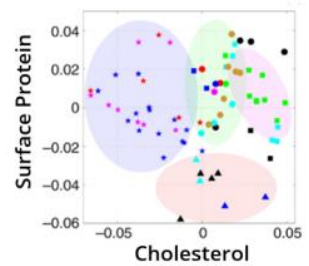

Figure 2: Representative data obtained by NTA, TRPS, and Raman methods. NTA provides (a) concentration versus size and (b) refractive index and size of individual EVs. TRPS provides (c) concentration versus size and (d) size and $\zeta$-potential of individual EVs. Raman provides (e) overall chemical content of the vesicle and (f) distributions of individual chemicals for individual EVs. (a) and (e) are adapted from Ref. ${ }^{47}$ under the terms of a CC BY-NC license, (b) is adapted from Ref. ${ }^{3}$ under the terms of a CC BY-NC license. (c) is adapted from Ref. ${ }^{34}$ under the terms of a CC BY-NC license. (d) is adapted with permission from Ref. ${ }^{60}$, Copyright 2012 American Chemical Society. (f) is adapted from Ref. ${ }^{48}$ under the terms of a CC BY-NC license. 


\section{References}

1. D'Souza-Schorey C, Di Vizio D. Biology and proteomics of extracellular vesicles: harnessing their clinical potential. Expert Review of Proteomics 2014;11(3):251-253.

2. van der Pol E, Coumans FAW, Grootemaat AE, Gardiner C, Sargent IL, Harrison P, Sturk A, van Leeuwen TG, Nieuwland R. Particle size distribution of exosomes and microvesicles determined by transmission electron microscopy, flow cytometry, nanoparticle tracking analysis, and resistive pulse sensing. Journal of Thrombosis and Haemostasis 2014;12(7):11821192.

3. Gardiner C, Shaw M, Hole P, Smith J, Tannetta D, Redman CW, Sargent IL. Measurement of refractive index by nanoparticle tracking analysis reveals heterogeneity in extracellular vesicles. J Extracell Vesicles 2014;3:25361.

4. Sodar BW, Kittel A, Paloczi K, Vukman KV, Osteikoetxea X, Szabo-Taylor K, Nemeth A, Sperlagh B, Baranyai T, Giricz Z and others. Low-density lipoprotein mimics blood plasma-derived exosomes and microvesicles during isolation and detection. Scientific Reports 2016;6.

5. Gyorgy B, Modos K, Pallinger E, Paloczi K, Pasztoi M, Misjak P, Deli MA, Sipos A, Szalai A, Voszka I and others. Detection and isolation of cell-derived microparticles are compromised by protein complexes resulting from shared biophysical parameters. Blood 2011;117(4):E39-E48.

6. Dragovic RA, Southcombe JH, Tannetta DS, Redman CW, Sargent IL. Multicolor flow cytometry and nanoparticle tracking analysis of extracellular vesicles in the plasma of normal pregnant and pre-eclamptic women. Biol Reprod 2013;89(6):151.

7. Wolf $\mathrm{P}$. The nature and significance of platelet products in human plasma. $\mathrm{Br}$ J Haematol 1967;13(3):269-88.

8. Heijnen HF, Schiel AE, Fijnheer R, Geuze HJ, Sixma JJ. Activated platelets release two types of membrane vesicles: microvesicles by surface shedding and exosomes derived from exocytosis of multivesicular bodies and alphagranules. Blood 1999;94(11):3791-3799.

9. Italiano JE, Mairuhu ATA, Flaumenhaft R. Clinical Relevance of Microparticles from Platelets and Megakaryocytes. Current opinion in hematology 2010;17(6):578-584.

10. Berckmans RJ, Nieuwland R, Boing AN, Romijn FP, Hack CE, Sturk A. Cellderived microparticles circulate in healthy humans and support low grade thrombin generation. Thromb.Haemost. 2001;85(4):639-646.

11. Robert S, Poncelet P, Lacroix R, Arnaud L, Giraudo L, Hauchard A, Sampol J, Dignat-George F. Standardization of platelet-derived microparticle counting using calibrated beads and a Cytomics FC500 routine flow cytometer: a first step towards multicenter studies? J.Thromb.Haemost. 2009;7(1):190-197.

12. Gyorgy B, Paloczi K, Kovacs A, Barabas E, Beko G, Varnai K, Pallinger E, Szabo-Taylor K, Szabo TG, Kiss AA and others. Improved circulating microparticle analysis in acid-citrate dextrose (ACD) anticoagulant tube. Thrombosis Research 2014;133(2):285-292. 
13. Arraud N, Linares R, Tan S, Gounou C, Pasquet JM, Mornet S, Brisson AR. Extracellular vesicles from blood plasma: determination of their morphology, size, phenotype and concentration. J Thromb Haemost 2014;12(5):614-27.

14. Stoner SA, Duggan E, Condello D, Guerrero A, Turk JR, Narayanan PK, Nolan JP. High sensitivity flow cytometry of membrane vesicles. Cytometry A 2016;89(2):196-206.

15. Chandler WL, Yeung W, Tait JF. A new microparticle size calibration standard for use in measuring smaller microparticles using a new flow cytometer. J.Thromb.Haemost. 2011;9(6):1216-1224.

16. Connor DE, Exner T, Ma DDF, Joseph JE. The majority of circulating plateletderived microparticles fail to bind annexin $\mathrm{V}$, lack phospholipid-dependent procoagulant activity and demonstrate greater expression of glycoprotein Ib. Thrombosis and haemostasis 2010;103(5):1044-1052.

17. Flaumenhaft R, Dilks JR, Richardson J, Alden E, Patel-Hett SR, Battinelli E, Klement GL, Sola-Visner M, Italiano JE, Jr. Megakaryocyte-derived microparticles: direct visualization and distinction from platelet-derived microparticles. Blood 2009;113(5):1112-1121.

18. Smalley DM, Root KE, Cho H, Ross MM, Ley K. Proteomic discovery of 21 proteins expressed in human plasma-derived but not platelet-derived microparticles. Thromb.Haemost. 2007;97(1):67-80.

19. Nurden AT. Platelets, inflammation and tissue regeneration. Thromb.Haemost. 2011;105 Suppl 1:S13-S33.

20. Hunter MP, Ismail N, Zhang X, Aguda BD, Lee EJ, Yu L, Xiao T, Schafer J, Lee ML, Schmittgen TD and others. Detection of microRNA expression in human peripheral blood microvesicles. PLoS One 2008;3(11):e3694.

21. Laffont B, Corduan A, Ple H, Duchez AC, Cloutier N, Boilard E, Provost P. Activated platelets can deliver mRNA regulatory Ago $2 *$ microRNA complexes to endothelial cells via microparticles. Blood 2013;122(2):253-61.

22. Laffont B, Corduan A, Rousseau M, Duchez AC, Lee CH, Boilard E, Provost P. Platelet microparticles reprogram macrophage gene expression and function. Thromb Haemost 2016;115(2):311-23.

23. Liang H, Yan X, Pan Y, Wang Y, Wang N, Li L, Liu Y, Chen X, Zhang CY, Gu H and others. MicroRNA-223 delivered by platelet-derived microvesicles promotes lung cancer cell invasion via targeting tumor suppressor EPB41L3. Mol Cancer 2015;14:58.

24. Saveyn H, De Baets B, Thas O, Hole P, Smith J, Van der Meeren P. Accurate particle size distribution determination by nanoparticle tracking analysis based on 2-D Brownian dynamics simulation. Journal of Colloid and Interface Science 2010;352(2):593-600.

25. van der Pol E, Coumans FAW, Sturk A, Nieuwland R, van Leeuwen TG. Refractive Index Determination of Nanoparticles in Suspension Using Nanoparticle Tracking Analysis. Nano Letters 2014;14(11):6195-6201.

26. Dragovic RA, Gardiner C, Brooks AS, Tannetta DS, Ferguson DJP, Hole P, Carr B, Redman CWG, Harris AL, Dobson PJ and others. Sizing and phenotyping of cellular vesicles using Nanoparticle Tracking Analysis. NanomedicineNanotechnology Biology and Medicine 2011;7(6):780-788. 
27. Gardiner C, Ferreira YJ, Dragovic RA, Redman CW, Sargent IL. Extracellular vesicle sizing and enumeration by nanoparticle tracking analysis. J Extracell Vesicles 2013;2.

28. Chen Y, Cai JY, Xu QC, Chen ZW. Atomic force bio-analytics of polymerization and aggregation of phycoerythrin-conjugated immunoglobulin $\mathrm{G}$ molecules. Molecular Immunology 2004;41(12):1247-1252.

29. Wang JJ, Zhong Y, Ma XT, Xiao X, Cheng CF, Chen YS, Iwuchukwu I, Gaines KJ, Zhao B, Liu SM and others. Analyses of Endothelial Cells and Endothelial Progenitor Cells Released Microvesicles by Using Microbead and Q-dot Based Nanoparticle Tracking Analysis. Scientific Reports 2016;6.

30. Sowerby SJ, Broom MF, Petersen GB. Dynamically resizable nanometre-scale apertures for molecular sensing. Sensors and Actuators B-Chemical 2007;123(1):325-330.

31. Roberts GS, Kozak D, Anderson W, Broom MF, Vogel R, Trau M. Tunable Nano/Micropores for Particle Detection and Discrimination: Scanning Ion Occlusion Spectroscopy. Small 2010;6(23):2653-2658.

32. Anderson W, Lane R, Korbie D, Trau M. Observations of Tunable Resistive Pulse Sensing for Exosome Analysis: Improving System Sensitivity and Stability. Langmuir 2015;31(23):6577-6587.

33. Maas SLN, de Vrij J, van der Vlist EJ, Geragousian B, van Bloois L, Mastrobattista E, Schiffelers RM, Wauben MHM, Broekman MLD, Nolte-'t Hoen ENM. Possibilities and limitations of current technologies for quantification of biological extracellular vesicles and synthetic mimics. Journal of Controlled Release 2015;200:87-96.

34. Coumans FA, van der Pol E, Boing AN, Hajji N, Sturk G, van Leeuwen TG, Nieuwland R. Reproducible extracellular vesicle size and concentration determination with tunable resistive pulse sensing. J Extracell Vesicles 2014;3:25922.

35. van der Pol E, Coumans F, Varga Z, Krumrey M, Nieuwland R. Innovation in detection of microparticles and exosomes. Journal of Thrombosis and Haemostasis 2013;11:36-45.

36. Burnouf T, Goubran HA, Chou ML, Devos D, Radosevic M. Platelet microparticles: Detection and assessment of their paradoxical functional roles in disease and regenerative medicine. Blood Reviews 2014;28(4):155166.

37. Lane RE, Korbie D, Anderson W, Vaidyanathan R, Trau M. Analysis of exosome purification methods using a model liposome system and tunableresistive pulse sensing. Scientific Reports 2015;5.

38. Hill AF, Pegtel DM, Lambertz U, Leonardi T, O'Driscoll L, Pluchino S, TerOvanesyan D, Nolte-'t Hoen EN. ISEV position paper: extracellular vesicle RNA analysis and bioinformatics. J Extracell Vesicles 2013;2.

39. Osteikoetxea X, Sodar B, Nemeth A, Szabo-Taylor K, Paloczi K, Vukman KV, Tamasi V, Balogh A, Kittel A, Pallinger E and others. Differential detergent sensitivity of extracellular vesicle subpopulations. Organic \& Biomolecular Chemistry 2015;13(38):9775-9782. 
40. Osteikoetxea X, Balogh A, Szabo-Taylor K, Nemeth A, Szabo TG, Paloczi K, Sodar B, Kittel A, Gyorgy B, Pallinger E and others. Improved Characterization of EV Preparations Based on Protein to Lipid Ratio and Lipid Properties. Plos One 2015;10(3).

41. Smith ZJ, Huser TR, Wachsmann-Hogiu S. Raman scattering in pathology. Analytical Cellular Pathology 2012;35(3):145-163.

42. Ajito K, Torimitsu K. Laser trapping and Raman spectroscopy of single cellular organelles in the nanometer range. Lab on a Chip 2002;2(1):11-14.

43. Xie CG, Dinno MA, Li YQ. Near-infrared Raman spectroscopy of single optically trapped biological cells. Optics Letters 2002;27(4):249-251.

44. Smith ZJ, Berger AJ. Validation of an integrated Raman- and angularscattering microscopy system on heterogeneous bead mixtures and single human immune cells. Applied Optics 2009;48(10):D109-D120.

45. Chan JW. Recent advances in laser tweezers Raman spectroscopy (LTRS) for label-free analysis of single cells. J Biophotonics 2013;6(1):36-48.

46. Lavialle F, Deshayes S, Gonnet F, Larquet E, Kruglik SG, Boisset N, Daniel R, Alfsen A, Tatischeff I. Nanovesicles released by Dictyostelium cells: A potential carrier for drug delivery. International Journal of Pharmaceutics 2009;380(1-2):206-215.

47. Tatischeff I, Larquet E, Falcon-Perez JM, Turpin PY, Kruglik SG. Fast characterisation of cell-derived extracellular vesicles by nanoparticles tracking analysis, cryo-electron microscopy, and Raman tweezers microspectroscopy. J Extracell Vesicles 2012;1.

48. Smith ZJ, Lee C, Rojalin T, Carney RP, Hazari S, Knudson A, Lam K, Saari H, Ibanez EL, Viitala T and others. Single exosome study reveals subpopulations distributed among cell lines with variability related to membrane content. J Extracell Vesicles 2015;4:28533.

49. Fleischmann M, Hendra PJ, Mcquilla.Aj. Raman-Spectra of Pyridine Adsorbed at a Silver Electrode. Chemical Physics Letters 1974;26(2):163-166.

50. Fan MK, Andrade GFS, Brolo AG. A review on the fabrication of substrates for surface enhanced Raman spectroscopy and their applications in analytical chemistry. Analytica Chimica Acta 2011;693(1-2):7-25.

51. Lee C, Zhang P. Facile synthesis of gelatin-protected silver nanoparticles for SERS applications. Journal of Raman Spectroscopy 2013;44(6):823-826.

52. Kerr LT, Gubbins L, Gorzel KW, Sharma S, Kell M, McCann A, Hennelly BM. Raman spectroscopy and SERS analysis of ovarian tumour derived exosomes (TEXs): a preliminary study. Biophotonics: Photonic Solutions for Better Health Care Iv 2014;9129.

53. Tirinato L, Gentile F, Di Mascolo D, Coluccio ML, Das G, Liberale C, Pullano SA, Perozziello G, Francardi M, Accardo A and others. SERS analysis on exosomes using super-hydrophobic surfaces. Microelectronic Engineering 2012;97:337-340.

54. Lee C, Carney RP, Hazari S, Smith ZJ, Knudson A, Robertson CS, Lam KS, Wachsmann-Hogiu S. 3D plasmonic nanobowl platform for the study of exosomes in solution. Nanoscale 2015;7(20):9290-9297. 
55. Stremersch S, Marro M, Pinchasik BE, Baatsen P, Hendrix A, De Smedt SC, Loza-Alvarez P, Skirtach AG, Raemdonck K, Braeckmans K. Identification of Individual Exosome-Like Vesicles by Surface Enhanced Raman Spectroscopy. Small 2016.

56. Im H, Shao HL, Park YI, Peterson VM, Castro CM, Weissleder R, Lee H. Labelfree detection and molecular profiling of exosomes with a nano-plasmonic sensor. Nature Biotechnology 2014;32(5):490-U219.

57. Zhu L, Wang K, Cui J, Liu H, Bu XL, Ma HL, Wang WZ, Gong H, Lausted C, Hood $\mathrm{L}$ and others. Label-Free Quantitative Detection of Tumor-Derived Exosomes through Surface Plasmon Resonance Imaging. Analytical Chemistry 2014;86(17):8857-8864.

58. Brolo AG. Plasmonics for future biosensors. Nature Photonics 2012;6(11):709-713.

59. Su J. Label-Free Single Exosome Detection Using Frequency-Locked Microtoroid Optical Resonators. Acs Photonics 2015;2(9):1241-1245.

60. Kozak D, Anderson W, Vogel R, Chen S, Antaw F, Trau M. Simultaneous size and zeta-potential measurements of individual nanoparticles in dispersion using size-tunable pore sensors. ACS Nano 2012;6(8):6990-7. 